Bull. Korean Math. Soc. 51 (2014), No. 5, pp. 1485-1502

http://dx.doi.org/10.4134/BKMS.2014.51.5.1485

\title{
ASYMPTOTIC PROPERTIES OF THE HYPERBOLIC METRIC ON THE SPHERE WITH THREE CONICAL SINGULARITIES
}

\author{
TANRAN ZHANG
}

\begin{abstract}
The explicit formula for the hyperbolic metric $\lambda_{\alpha, \beta, \gamma}(z)|d z|$ on the thrice-punctured sphere $\mathbb{P} \backslash\{0,1, \infty\}$ with singularities of order $0<\alpha, \beta<1, \gamma \leq 1, \alpha+\beta+\gamma>2$ at $0,1, \infty$ was given by Kraus, Roth and Sugawa in [9]. In this article we investigate the asymptotic properties of the higher order derivatives of $\lambda_{\alpha, \beta, \gamma}(z)$ near the origin and give more precise descriptions for the asymptotic behavior of $\lambda_{\alpha, \beta, \gamma}(z)$.
\end{abstract}

\section{Introduction}

The hyperbolic metric, also called the Poincaré metric, plays an important role in geometric function theory. Suppose that $X$ is a hyperbolic Riemann surface. A smooth conformal metric $\lambda(z)|d z|$ on $X$ is the hyperbolic metric if and only if it is complete and its Gaussian curvature $\kappa_{\lambda}=(\Delta \log \lambda) / \lambda^{2}$ is a negative constant (see [5, Theorem 5.9], and [14]). In this article we normalize the curvature to be -4 for the hyperbolic metric. In particular, if $X$ is compact, the hyperbolic metric on $X$ is characterized only by $\kappa_{\lambda} \equiv-4$. What happens if $\lambda(z)|d z|$ admits finitely many singularities on a compact Riemann surface $X$ ? Due to Nitsche [11], if the conformal metric $\lambda(z)|d z|$ of the curvature -4 on $X$ has an isolated singularity at a point $p_{0}$ with $z\left(p_{0}\right)=z_{0}$ for the local coordinate $z$, the limit

$$
\alpha=\lim _{r \rightarrow 0} \frac{\sup _{\left|z-z_{0}\right|=r} \log \lambda(z)}{-\log r},
$$

exists and $\alpha \leq 1$ (see also [7]). Thus the following problem for the existence and uniqueness comes up: Does there exist a complete conformal metric of constant curvature -4 on the compact Riemann surface $X$, with the prescribed singularities $p_{j} \in X$ of order $\alpha_{j}, j=1,2, \ldots$, and is it unique if it exists? Heins [4] proved that, for $X$ of genus $g$ and singularities $p_{1}, \ldots, p_{N} \in X$ of orders $\alpha_{1}, \ldots, \alpha_{N}$, such a metric exists if and only if $2 g-2+\sum_{j=1}^{N} \alpha_{j}>0$, and it

Received July 22, 2013; Revised January 23, 2014.

2010 Mathematics Subject Classification. 30L10, 33D15.

Key words and phrases. conical singularities, hyperbolic metrics, special functions.

(C)2014 Korean Mathematical Society 
is unique if it exists. This metric is called the generalized hyperbolic metric. As a spacial case of Heins' result, Picard [12] gave a result for $X$ of genus 0. Troyanov [13] discussed conditions under which a function on a Riemann surface is the Gaussian curvature of some conformal metric with a prescribed set of singularities of conical type.

Generally, an explicit formula for the hyperbolic metric on a hyperbolic plane domain is hard to find and by now it is known explicitly only for a few cases. Nitsche [11] studied the asymptotic behavior of the hyperbolic metric near an isolated singularity. Kraus and Roth [7] extended Nitsche's work to a conformal metric $\lambda(z)|d z|$ with negative, Hölder continuous curvature function $\kappa(z)$ near the singularity. They estimated the first and second order derivatives of $\log \lambda(z)$ near the origin. The author [16] further complemented their results by giving the estimates for higher order derivatives. It is important to verify the sharpness of the estimates, and furthermore, to consider more precise information for the asymptotic properties of $\log \lambda(z)$. Thus we investigate a special conformal metric. According to Picard's result [12], the maximal hyperbolic plane domain is the thrice-punctured Riemann sphere $\widehat{\mathbb{C}} \backslash\left\{z_{1}, z_{2}, z_{3}\right\}$. Therefore the generalized hyperbolic metric on $\widehat{\mathbb{C}} \backslash\left\{z_{1}, z_{2}, z_{3}\right\}$ has its own significance since it helps to determine the precise asymptotic properties near a singularity. In the (normalized) thrice-punctured Riemann sphere $\widehat{\mathbb{C}} \backslash\{0,1, \infty\}$ with singularities of orders $\alpha, \beta$, $\gamma$ at $0,1, \infty$, respectively, where $0<\alpha, \beta<1$, $\gamma \leq 1, \alpha+\beta+\gamma>2$, Kraus, Roth, and Sugawa [9] gave an explicit formula for the hyperbolic metric $\lambda_{\alpha, \beta, \gamma}(z)|d z|$ in terms of some special functions. In this article, we will examine sharpness of the estimates given in [7] and [16], by using the generalized hyperbolic metric $\lambda_{\alpha, \beta, \gamma}(z)|d z|$ near the origin. We will further show that certain limits exist, which is stronger than order estimates (see Theorems 3.4 and 4.1).

In 1997, Minda [10] studied the behavior of the hyperbolic metric in a neighborhood of a puncture on the plane domain using the uniformisation theorem for up to second order derivatives. His results can be extended to higher order derivatives of a conformal metric with negative curvature on an arbitrary hyperbolic region (see [16]). However, if the order $\alpha<1$, this kind of limit $l$ may depend not only on $\alpha$, but also on the shape of the domain, or the properties of the curvature function. In this article, we will observe it by examining the case of the generalized hyperbolic metric $\lambda_{\alpha, \beta, \gamma}(z)$.

\section{Preliminaries}

For complex numbers $a, b, c$ with $c \neq 0,-1,-2, \ldots$, the Gaussian hypergeometric function is defined as

$$
F(a, b, c ; z)=\sum_{n=0}^{\infty} \frac{(a)_{n}(b)_{n}}{(c)_{n}} \frac{z^{n}}{n !}, \quad|z|<1,
$$


where $(a)_{n}$ is the Pochhammer symbol, namely, $(a)_{0}=1$ and $(a)_{n}=a(a+$ 1) $\cdots(a+n-1)$ for $n=1,2,3, \ldots$ It is continued analytically to the slit plane $\mathbb{C} \backslash[1,+\infty)$. Its derivative is given by

$$
\frac{d}{d z} F(a, b, c ; z)=\frac{a b}{c} F(a+1, b+1, c+1 ; z) .
$$

We can immediately obtain

$$
\frac{d^{n}}{d z^{n}} F(a, b, c ; z)=\frac{(a)_{n}(b)_{n}}{(c)_{n}} F(a+n, b+n, c+n ; z) .
$$

We have

$$
\begin{aligned}
& F(a, b, c ; z) \\
= & \frac{\Gamma(c) \Gamma(c-a-b)}{\Gamma(c-a) \Gamma(c-b)} F(a, b, a+b-c+1 ; 1-z) \\
& +(1-z)^{c-a-b} \frac{\Gamma(c) \Gamma(a+b-c)}{\Gamma(a) \Gamma(b)} F(c-a, c-b, c-a-b+1 ; 1-z)
\end{aligned}
$$

for $|\arg (1-z)|<\pi$, where $\Gamma(z)$ is Gamma function (see $[1,15.3 .6])$. Each term of (2.3) has a singularity at $z=1$ when $c=a+b+n, n=0,1,2, \ldots$, and this case is covered by

$$
\begin{aligned}
& F(a, b, a+b+n ; z) \\
= & \frac{\Gamma(n) \Gamma(a+b+n)}{\Gamma(a+n) \Gamma(b+n)} \sum_{j=0}^{n-1} \frac{(a)_{j}(b)_{j}}{j !(1-n)_{j}}(1-z)^{j} \\
& -\frac{\Gamma(a+b+n)}{\Gamma(a) \Gamma(b)}(z-1)^{n} \sum_{j=0}^{\infty} \frac{(a+n)_{j}(b+n)_{j}}{j !(j+n) !}(1-z)^{j}[\log (1-z) \\
& -\Psi(j+1)-\Psi(j+n+1)+\Psi(a+j+n)+\Psi(b+j+n)]
\end{aligned}
$$

for $|\arg (1-z)|<\pi,|1-z|<1$, where $\Psi(z)=\Gamma^{\prime}(z) / \Gamma(z)$ is the digamma function (see $[1,15.3 .11]$ ). We take the convention that $\sum_{j=a}^{b}=0$ if $b<a$ here and after. The behavior of the hypergeometric function near $z=1$ satisfies

$$
\left\{\begin{array}{l}
F(a, b, c ; 1)=\frac{\Gamma(c) \Gamma(c-a-b)}{\Gamma(c-a) \Gamma(c-b)}, \text { if } a+b<c, \\
F(a, b, a+b ; z)=\frac{1}{B(a, b)}\left(\log \frac{1}{1-z}+R(a, b)\right)(1+O(1-z)), \\
F(a, b, c ; z)=(1-z)^{c-a-b} F(c-a, c-b, c ; z), \text { if } a+b>c,
\end{array}\right.
$$

where

$$
B(a, b)=\frac{\Gamma(a) \Gamma(b)}{\Gamma(a+b)}
$$

is Beta function and

$$
R(a, b)=2 \Psi(1)-\Psi(a)-\Psi(b) .
$$


The asymptotic formula in (2.5) for the case $a+b=c$ is due to Ramanujan (see $[1,2])$.

In the domain $\Omega \subseteq \mathbb{C}$, every continuous function $\lambda: \Omega \rightarrow(0,+\infty)$ induces a conformal metric, denoted by $\lambda(z)|d z|$, on $\Omega$ (see $[4,8]$ ). We call $\lambda(z)$ the density function of the metric $\lambda(z)|d z|$. Suppose that $\Omega$ is a hyperbolic plane domain. For a point $p \in \Omega$, let $z$ be local coordinates such that $z(p)=0$. We say a conformal metric $\lambda(z)|d z|$ on the punctured domain $\Omega^{*}:=\Omega \backslash\{p\}$ has a conical singularity of order $\alpha \leq 1$ at the point $p$, if, in local coordinates $z$,

$$
\log \lambda(z)= \begin{cases}-\alpha \log |z|+v(z) & \text { if } \quad \alpha<1 \\ -\log |z|-\log \log (1 /|z|)+w(z) & \text { if } \quad \alpha=1\end{cases}
$$

where $v(z), w(z)=O(1)$ as $z(p) \rightarrow 0$ with $O$ and $o$ being the Landau symbols in this article. For $u(z):=\log \lambda(z)$, the order $\alpha$ of $\lambda(z)|d z|$ at the conical singularity $p$ is again the order of $u(z)$ at the conical singularity $\log p$. We call the point $p$ a corner of order $\alpha$ if $\alpha<1$ and a cusp if $\alpha=1$. It is evident that the cusp is the limit case of a corner.

A conformal metric $\lambda(z)|d z|$ on a domain $\Omega \subseteq \mathbb{C}$ is regular, if its density $\lambda(z)$ is positive and twice continuously differentiable on $G$, i.e., $\lambda(z) \in C^{2}(G)$. The Gaussian curvature $\kappa_{\lambda}(z)$ of a regular conformal metric $\lambda(z)|d z|$ is defined by

$$
\kappa_{\lambda}(z)=-\frac{\Delta \log \lambda(z)}{\lambda(z)^{2}}, \quad \text { where } \Delta=\frac{\partial^{2}}{\partial x^{2}}+\frac{\partial^{2}}{\partial y^{2}}, \quad z=x+i y .
$$

A very basic property of Gaussian curvature is the conformal invariance (see e.g. [5]).

The hyperbolic metric of the Gaussian curvature -4 on the unit disk $\mathbb{D}$ is given by

$$
\lambda_{\mathbb{D}}(z)|d z|=\frac{|d z|}{1-|z|^{2}} .
$$

The hyperbolic metric $\lambda_{\Omega}(w)|d w|$ on a hyperbolic domain $\Omega$ is defined by

$$
\lambda_{\Omega}(w)|d w|=\frac{\lambda_{\mathbb{D}}(z)}{\pi^{\prime}(z) \mid}|d w|,
$$

where $\pi: \mathbb{D} \rightarrow \Omega$ is the universal covering projection, $\pi(z)=\omega$. The following result gives the explicit formula for the generalized hyperbolic metric on $\widehat{\mathbb{C}} \backslash\{0,1, \infty\}$. The terminology generalized hyperbolic metric is motivated by the fact that if all singularities are cusps, then we get back the standard hyperbolic metric on the punctured sphere $\widehat{\mathbb{C}} \backslash\left\{z_{1}, \ldots, z_{n}\right\}$ (see [9]).

Theorem A ([10]). Let $0<\alpha, \beta<1$ and $0<\gamma \leq 1$ satisfying $\alpha+\beta+$ $\gamma>2$. Then the generalized hyperbolic density on the thrice-punctured sphere $\widehat{\mathbb{C}} \backslash\{0,1, \infty\}$ of orders $\alpha, \beta, \gamma$ at $0,1, \infty$, respectively, can be expressed by

$$
\lambda_{\alpha, \beta, \gamma}(z)
$$




$$
\begin{aligned}
& =\frac{1}{|z|^{\alpha}|1-z|^{\beta}} \cdot \frac{K_{3}}{K_{1}\left|\varphi_{1}(z)\right|^{2}+K_{2}\left|\varphi_{2}(z)\right|^{2}+2 \operatorname{Re}\left(\varphi_{1}(z) \varphi_{2}(\bar{z})\right)} \\
& =\frac{1}{|z|^{\alpha}|1-z|^{\beta}} \cdot \frac{\delta(1-\alpha)}{\left|\varphi_{1}(z)\right|^{2}-\delta^{2}|1-z|^{2-2 \alpha}\left|\varphi_{3}(z)\right|^{2}}
\end{aligned}
$$

in the twice-punctured plane $\mathbb{C} \backslash\{0,1\}$, where

$$
\begin{aligned}
K_{1} & :=-\frac{\Gamma(c-a) \Gamma(c-b)}{\Gamma(c) \Gamma(c-a-b)}, \quad K_{2}:=-\frac{\Gamma(a+1-c) \Gamma(b+1-c)}{\Gamma(1-c) \Gamma(a+b+1-c)}, \\
K_{3} & :=\sqrt{\frac{\sin (\pi a) \sin (\pi b)}{\sin (\pi(c-a)) \sin (\pi(c-b))}} \cdot \frac{\Gamma(a+b+1-c) \Gamma(c)}{\Gamma(a) \Gamma(b)}
\end{aligned}
$$

and

$$
\begin{gathered}
\varphi_{1}(z)=F(a, b, c ; z), \quad \varphi_{2}(z)=F(a, b, a+b-c+1 ; 1-z), \\
\varphi_{3}(z)=F(a-c+1, b-c+1,2-c ; z)
\end{gathered}
$$

with

$$
\begin{gathered}
a=\frac{\alpha+\beta-\gamma}{2}, b=\frac{\alpha+\beta+\gamma-2}{2}, c=\alpha \\
\delta=\frac{\Gamma(c)}{\Gamma(2-c)}\left(\frac{\Gamma(1-a) \Gamma(1-b) \Gamma(a+1-c) \Gamma(b+1-c)}{\Gamma(a) \Gamma(b) \Gamma(c-a) \Gamma(c-b)}\right)^{1 / 2} .
\end{gathered}
$$

The Gaussian curvature of $\lambda_{\alpha, \beta, \gamma}(z)$ defined by (2.9) and (2.10) is -4 . Note that $\varphi_{1}$ and $\varphi_{3}$ are analytic in $\mathbb{C} \backslash[1,+\infty), \varphi_{2}$ is analytic in $\mathbb{C} \backslash(-\infty, 0]$.

We denote $\lambda(z)=\lambda_{\alpha, \beta, \gamma}(z)$ briefly in the rest of this article. The following theorem is due to Kraus, Roth and Sugawa [9]. They did not give the explicit formula of (2.14), but it is easy to deduce it from (2.10).

Theorem B. Let $0<\alpha<1$. Then for the hyperbolic density $\lambda(z)$ given in (2.10), we have

$$
\lim _{z \rightarrow 0}|z|^{\alpha} \lambda(z)=\frac{\delta}{1-\delta^{2}}(1-\alpha)
$$

where $\delta$ is as in (2.13), $a, b$ and $c$ are as in (2.12).

\section{Case $0<\alpha<1$}

In this section we focus on the hyperbolic metric $\lambda(z)|d z|$ when $0<\alpha<1$. We can consider the asymptotic behavior of $\lambda(z)$ only near the origin. By the expression of $\lambda(z)$, we know that the singularity $z=1$ is in the same situation as the origin. As for the infinity, we can change the coordinates by a conformal function, say, $z \mapsto 1 / z$, to map $\infty$ to 0 . So it is convenient to consider the case near the origin. In expression (2.9), for orders $0<\alpha, \beta<1$ and $0<\gamma \leq 1$, the real parameters $a, b, c$ given by condition (2.12) satisfy

$$
-\frac{1}{2}<a<1,-1<b<\frac{1}{2}, 0<c<1 .
$$


Denote

$$
\partial^{n}:=\frac{\partial^{n}}{\partial z^{n}}, \bar{\partial}^{n}:=\frac{\partial^{n}}{\partial \bar{z}^{n}}
$$

for $n \geq 1$. At first we give a lemma for the future use.

Lemma 3.1. For $\lambda(z)$ as in (2.9) with order $\alpha \in(0,1)$, let

$$
\begin{aligned}
M(z): & =K_{1}\left|\varphi_{1}(z)\right|^{2}+K_{2}\left|\varphi_{2}(z)\right|^{2}+2 \operatorname{Re}\left(\varphi_{1}(z) \varphi_{2}(\bar{z})\right) \\
& =\left(K_{1} \varphi_{1}(\bar{z})+\varphi_{2}(\bar{z})\right) \varphi_{1}(z)+\left(K_{2} \varphi_{2}(\bar{z})+\varphi_{1}(\bar{z})\right) \varphi_{2}(z) .
\end{aligned}
$$

Then for $a, b$ and $c$ defined in (2.12), $K_{1}$ and $K_{2}$ given by (2.11),

(i) $\lim _{z \rightarrow 0} \partial M(z)=\frac{a b}{c}\left(K_{1}-\frac{1}{K_{2}}\right)$ for $0<\alpha<1 / 2$,

(ii) $\partial M(z)=2 a b\left(K_{1}-\frac{1}{K_{2}}\right)+2 K_{2} Y(a, b, c) \frac{\bar{z}}{|z|}+O\left(|z|^{\frac{1}{2}}\right)$ for $\alpha=1 / 2$,

(iii) $\lim _{z \rightarrow 0} z^{n}|z|^{2 \alpha-2} \partial^{n} M(z)=\frac{(-1)^{n-1}(c)_{n-1} K_{2} Y(a, b, c)}{1-c}$ for $n \geq 2$ if $0<\alpha \leq$ $1 / 2$ and $n \geq 1$ if $1 / 2<\alpha<1$,

(iv) $\lim _{z \rightarrow 0} \bar{z}^{m} z^{n}|z|^{2 \alpha-2} \bar{\partial}^{m} \partial^{n} M(z)=(-1)^{n+m}(c)_{n-1}(c)_{m-1} K_{2} Y(a, b, c)$ for $m$, $n \geq 1$, where

$$
Y(a, b, c)=\left(\frac{\Gamma(c) \Gamma(a+b-c+1)}{\Gamma(a) \Gamma(b)}\right)^{2} .
$$

Remark. The relation (ii) can be expressed by $\partial M(z)=O(1)$. It is easy to see that there is no non-vanishing limit such as in (iii) for $n=1$ and $\alpha=1 / 2$.

Proof of Lemma 3.1. Since $\varphi_{1}(z), \varphi_{2}(z)$ are analytic in $\mathbb{C} \backslash[1,+\infty), \mathbb{C} \backslash(-\infty, 0]$, respectively, we have $\overline{\partial^{n} \varphi_{1}(z)}=\bar{\partial}^{n}\left(\varphi_{1}(\bar{z})\right)$ for $z \in \mathbb{C} \backslash[1,+\infty)$, and $\overline{\partial^{n} \varphi_{2}(z)}=$ $\bar{\partial}^{n}\left(\varphi_{2}(\bar{z})\right)$ for $z \in \mathbb{C} \backslash(-\infty, 0]$. For the limit in (i), we have

$$
\partial M(z)=\left(K_{1} \varphi_{1}(\bar{z})+\varphi_{2}(\bar{z})\right) \partial \varphi_{1}(z)+\left(K_{2} \varphi_{2}(\bar{z})+\varphi_{1}(\bar{z})\right) \partial \varphi_{2}(z) .
$$

From the property $(2.2)$,

$$
\partial \varphi_{1}(0)=\frac{a b}{c}
$$

By $(2.5)$,

$$
\varphi_{2}(0)=F(a, b, a+b-c+1 ; 1)=-\frac{1}{K_{2}}, \varphi_{1}(0)=1,
$$

provided that $a+b<a+b-c+1$. Thus

$$
K_{1} \varphi_{1}(0)+\varphi_{2}(0)=K_{1}-K_{2}^{-1} .
$$

Now we consider the term $\left(K_{2} \varphi_{2}(\bar{z})+\varphi_{1}(\bar{z})\right) \partial \varphi_{2}(z)$, which satisfies

$$
\lim _{z \rightarrow 0}\left(K_{2} \varphi_{2}(z)+\varphi_{1}(z)\right)=0 .
$$

Note that

$$
\varphi_{2}(z)=F(a, b, a+b-c+1 ; 1-z)
$$




$$
\begin{aligned}
= & \frac{\Gamma(a+b-c+1) \Gamma(1-c)}{\Gamma(b-c+1) \Gamma(a-c+1)} F(a, b, c ; z) \\
& +z^{1-c} \sqrt{Y(a, b, c)} F(b-c+1, a-c+1,2-c ; z) \\
= & -\frac{1}{K_{2}} \varphi_{1}(z)+z^{1-c} \sqrt{Y(a, b, c)} F(b-c+1, a-c+1,2-c ; z)
\end{aligned}
$$

for $|\arg (z)|<\pi$, which means $\varphi_{1}(z)$ and $\varphi_{2}(z)$ are related. Hence

(3.6) $K_{2} \varphi_{2}(z)+\varphi_{1}(z)=\frac{-K_{2} z^{1-c}}{1-c} \sqrt{Y(a, b, c)} F(b-c+1, a-c+1,2-c ; z)$

and

$$
\lim _{z \rightarrow 0} \frac{K_{2} \varphi_{2}(z)+\varphi_{1}(z)}{z^{1-c}}=\frac{-K_{2}}{1-c} \sqrt{Y(a, b, c)} .
$$

Near the origin, by (2.2), for $n \geq 1$,

$$
\begin{aligned}
\partial^{n} \varphi_{2}(z) & =\overline{\bar{\partial}^{n} \varphi_{2}(\bar{z})} \\
& =\frac{(a)_{n}(b)_{n}}{(a+b-c+1)_{n}}(-1)^{n} F(a+n, b+n, a+b-c+1+n ; 1-z) .
\end{aligned}
$$

By property (2.3), we have

$$
\begin{aligned}
& F(a+n, b+n, a+b-c+1+n ; 1-z) \\
= & \frac{\Gamma(a+b-c+1+n) \Gamma(1-c-n)}{\Gamma(b-c+1) \Gamma(a-c+1)} F(a+n, b+n, c+n ; z) \\
& +z^{1-c-n} \frac{\Gamma(a+b-c+1+n) \Gamma(c+n-1)}{\Gamma(a+n) \Gamma(b+n)} F(b-c+1, a-c+1,2-c-n ; z)
\end{aligned}
$$

for $|\arg (z)|<\pi$. Then near the origin, substituting the expression above into (3.8), we have

$$
\begin{aligned}
& \partial^{n} \varphi_{2}(z) \\
= & \frac{(a)_{n}(b)_{n}}{(a+b-c+1)_{n}}(-1)^{n} \frac{\Gamma(a+b-c+1+n) \Gamma(1-c-n)}{\Gamma(b-c+1) \Gamma(a-c+1)} F(a+n, b+n, c+n ; z) \\
& +\frac{(-1)^{n}}{z^{n+c-1}} \frac{\Gamma(a+b-c+1) \Gamma(c+n-1)}{\Gamma(a) \Gamma(b)} F(b-c+1, a-c+1,2-c-n ; z),
\end{aligned}
$$

which leads to the limit

$$
\lim _{z \rightarrow 0} z^{n+c-1} \partial^{n} \varphi_{2}(z)=(-1)^{n}(c)_{n-1} \sqrt{Y(a, b, c)} .
$$

Letting $n=1$ in (3.9) and combining with (3.6), we have

$$
\lim _{z \rightarrow 0}\left(K_{2} \varphi_{2}(\bar{z})+\varphi_{1}(\bar{z})\right) \partial \varphi_{2}(z)=0
$$


if $0<c=\alpha<\frac{1}{2}$. Thus

$$
\lim _{z \rightarrow 0} \partial M(z)=\lim _{z \rightarrow 0}\left(K_{1} \varphi_{1}(\bar{z})+\varphi_{2}(\bar{z})\right) \partial \varphi_{1}(z)=\frac{a b}{c}\left(K_{1}-\frac{1}{K_{2}}\right)
$$

provided (3.3) and (3.5).

We note that (3.6) and (3.9) are still valid for $n=1, \alpha=1 / 2$, and combining with (3.3) and (3.4), the relation (ii) hold.

For the relation (iii), at first we have

$$
\partial^{n} M(z)=\left(K_{1} \varphi_{1}(\bar{z})+\varphi_{2}(\bar{z})\right) \partial^{n} \varphi_{1}(z)+\left(K_{2} \varphi_{2}(\bar{z})+\varphi_{1}(\bar{z})\right) \partial^{n} \varphi_{2}(z) .
$$

From the property $(2.2)$,

$$
\partial^{n} \varphi_{1}(0)=\frac{(a)_{n}(b)_{n}}{(c)_{n}}
$$

for $n \geq 1$. Since $n>2 \alpha-2$ for all $n \geq 2$ and $0<\alpha<1$, from (3.12) and (3.5), we know that the limit in (iii) is only determined by the term $\left(K_{2} \varphi_{2}(\bar{z})+\right.$ $\left.\varphi_{1}(\bar{z})\right) \partial^{n} \varphi_{2}(z)$. Combining with (3.11), (3.10) and (3.7), we have

$$
\begin{aligned}
& \lim _{z \rightarrow 0} z^{n}|z|^{2 \alpha-2} \partial^{n} M(z) \\
= & \lim _{z \rightarrow 0} z^{n}|z|^{2 \alpha-2}\left(K_{2} \varphi_{2}(\bar{z})+\varphi_{1}(\bar{z})\right) \partial^{n} \varphi_{2}(z) \\
= & \lim _{z \rightarrow 0} \frac{K_{2} \varphi_{2}(\bar{z})+\varphi_{1}(\bar{z})}{\bar{z}^{1-\alpha}} \frac{z^{n}}{z^{1-\alpha}} \partial^{n} \varphi_{2}(z) \\
= & \lim _{z \rightarrow 0} \frac{K_{2} \varphi_{2}(\bar{z})+\varphi_{1}(\bar{z})}{\bar{z}^{1-c}} \cdot \lim _{z \rightarrow 0} z^{n+c-1} \partial^{n} \varphi_{2}(z) \\
= & \frac{(-1)^{n-1}(c)_{n-1} K_{2}}{1-c} Y(a, b, c)
\end{aligned}
$$

as in the relation (iii).

For the relation (iv), if $m \geq 1, n \geq 1$, we have

$$
\begin{aligned}
& \bar{\partial}^{m} \partial^{n} M(z) \\
= & \left(K_{1} \bar{\partial}^{m} \varphi_{1}(\bar{z})+\bar{\partial}^{m} \varphi_{2}(\bar{z})\right) \partial^{n} \varphi_{1}(z)+\left(K_{2} \bar{\partial}^{m} \varphi_{2}(\bar{z})+\bar{\partial}^{m} \varphi_{1}(\bar{z})\right) \partial^{n} \varphi_{2}(z) .
\end{aligned}
$$

Since $\lim _{z \rightarrow 0} z^{n+c-1} \partial^{n} \varphi_{1}(z)=0$, we can obtain

$$
\begin{aligned}
& \lim _{z \rightarrow 0} \bar{z}^{m} z^{n}|z|^{2 \alpha-2} \bar{\partial}^{m} \partial^{n} M(z) \\
= & \lim _{z \rightarrow 0} \frac{\bar{z}^{m} z^{n}}{|z|^{2-2 c}} K_{2} \bar{\partial}^{m} \varphi_{2}(\bar{z}) \partial^{n} \varphi_{2}(z) \\
= & \lim _{z \rightarrow 0} K_{2} \frac{\bar{z}^{m}}{\bar{z}^{1-c}} \bar{\partial}^{m} \varphi_{2}(\bar{z}) \cdot \frac{z^{n}}{z^{1-c}} \partial^{n} \varphi_{2}(z) \\
= & (-1)^{m+n}(c)_{m-1}(c)_{n-1} K_{2} Y(a, b, c)
\end{aligned}
$$

as in the relation (iv).

We denote $u(z):=\log \lambda(z)$ in the rest of this article. There are also some limits for $u(z)$ when $z$ is tending to the origin. 
Theorem 3.2. For $u(z)$ defined as above, if the order $\alpha \in(0,1)$, for $m, n \geq 1$ and $Y(a, b, c)$ as in $(3.2)$, we have

(i) $\lim _{z \rightarrow 0} z^{n} \partial^{n} u(z)=\frac{\alpha}{2}(-1)^{n}(n-1) !=\lim _{z \rightarrow 0} \bar{z}^{n} \bar{\partial}^{n} u(z)$,

(ii) $\lim _{z \rightarrow 0} \bar{z}^{m} z^{n}|z|^{2 \alpha-2} \bar{\partial}^{m} \partial^{n} u(z)=\frac{(-1)^{n+m}(c)_{n-1}(c)_{m-1} K_{2}^{2} Y(a, b, c)}{K_{1} K_{2}-1}$.

Proof. We note that

$$
u(z)=-\alpha \log |z|-\beta \log |1-z|+\log K_{3}-\log M(z)
$$

with $M(z)$ as in (3.1). At first we consider $\partial^{n} \log M(z)$. By (3.4),

$$
M(0)=K_{1} \varphi_{1}(0)+\varphi_{2}(0)=K_{1}-\frac{1}{K_{2}} .
$$

Then $M(0)>0$ for $a, b, c$ given by (2.12). When $0<\alpha<1$, from Lemma 3.1 we have

$$
\lim _{z \rightarrow 0} z^{k} \partial^{k} M(z)=0
$$

for $k \geq 1$. Observe that $\partial^{n} \log M(z)$ is the linear combination of products of $\partial^{k} M / M$ with $k \leq n$, then for $n \geq 1$, (3.14) leads to $\lim _{z \rightarrow 0} z^{n} \partial^{n} \log M(z)=0$. Since

$$
\partial^{n} \log |1-z|=-\frac{(n-1) !}{2(1-z)^{n}}, \quad \partial^{n} \log |z|=\frac{(-1)^{n-1}(n-1) !}{2 z^{n}},
$$

the first equality in (i) holds.

The second equality of (i) is obtained if we note that $u(z)$ is real-valued, and then

$$
\lim _{z \rightarrow 0} \bar{z}^{n} \bar{\partial}^{n} u(z)=\lim _{z \rightarrow 0} \overline{z^{n} \partial^{n} u(z)}=\frac{\alpha}{2}(-1)^{n}(n-1) ! .
$$

Now we discuss the term $\bar{\partial}^{m} \partial^{n} \log M(z)$ to prove the relation (ii). Since $\bar{\partial}^{m} \partial^{n} \log M(z)$ is the linear combination of products of $\bar{\partial}^{t} \partial^{k} M / M$ with $0 \leq$ $t \leq m, 0 \leq k \leq n$, Lemma 3.1 implies that

$$
\lim _{z \rightarrow 0} \bar{z}^{m} z^{n}|z|^{2 \alpha-2} \prod_{j=2}^{N} \frac{\bar{\partial}^{t_{j}} \partial^{k_{j}} M(z)}{M(z)}=0,
$$

where $2 \leq N \leq m+n, 1 \leq t_{j} \leq m$ and $1 \leq k_{j} \leq n$ for every index $j, 2 \leq j \leq N$. Thus

$$
\begin{aligned}
& \lim _{z \rightarrow 0} \bar{z}^{m} z^{n}|z|^{2 \alpha-2} \bar{\partial}^{m} \partial^{n} \log M(z) \\
= & \lim _{z \rightarrow 0} \bar{z}^{m} z^{n}|z|^{2 \alpha-2} \frac{\bar{\partial}^{m} \partial^{n} M(z)}{M(z)} \\
= & \frac{(-1)^{n+m}(c)_{n-1}(c)_{m-1} K_{2}^{2}}{K_{1} K_{2}-1} Y(a, b, c) .
\end{aligned}
$$

Note that $\bar{\partial}^{m} \partial^{n} \log |1-z|=0, \bar{\partial}^{m} \partial^{n} \log |z|=0$, therefore (ii) holds. 
Remark. The relation (i) of Theorem 3.2 is a specific version of (i) in Theorem 4.1 [16], and here we proved it in a different way. However, the relation (ii) of Theorem 3.2 is stronger than (ii) of Theorem 4.1 in [16].

For the hyperbolic metric, the following result corresponding to Theorem 1.2 in [16] holds.

Theorem 3.3. For $m, n \geq 0,0<\alpha<1$ and $\lambda(z)$ as in (2.9), the limit

$$
l_{m, n}:=\frac{1}{m ! n !} \lim _{z \rightarrow 0}|z|^{\alpha} \bar{z}^{m} z^{n} \bar{\partial}^{m} \partial^{n} \lambda(z)
$$

exists, and

$$
l_{m, n}=\left(\begin{array}{c}
-\frac{\alpha}{2} \\
n
\end{array}\right)\left(\begin{array}{c}
-\frac{\alpha}{2} \\
m
\end{array}\right) l_{0,0}
$$

where

$$
\left(\begin{array}{l}
\tau \\
j
\end{array}\right)=\frac{\tau(\tau-1) \cdots(\tau-j+1)}{j !}
$$

is the binomial coefficient.

Proof. By Theorem B,

$$
l_{0,0}=\lim _{z \rightarrow 0}|z|^{\alpha} \lambda(z)=\frac{\delta}{1-\delta^{2}}(1-\alpha) .
$$

Since

$$
\partial \lambda(z)=\lambda(z) \partial u(z)
$$

we have

$$
\partial^{n} \lambda(z)=\sum_{j=0}^{n-1}\left(\begin{array}{c}
n-1 \\
j
\end{array}\right) \partial^{n-j} u(z) \partial^{j} \lambda(z)
$$

by induction, where $\partial^{0} \lambda(z)=\bar{\partial}^{0} \lambda(z)=\lambda(z)$. Then

$$
l_{0, n}=\frac{1}{n !} \lim _{z \rightarrow 0} \sum_{j=0}^{n-1}\left(\begin{array}{c}
n-1 \\
j
\end{array}\right) z^{n-j} \partial^{n-j} u(z) \cdot|z|^{\alpha} z^{j} \partial^{j} \lambda(z) .
$$

From (i) of Theorem 3.2, $\lim _{z \rightarrow 0} z^{n-j} \partial^{n-j} u(z)$ exists, then $l_{0, n}$ exists. When $m=0, n=1,(3.15)$ and (3.16) lead to

$$
l_{0,1}=\lim _{z \rightarrow 0}|z|^{\alpha} z \partial \lambda(z)=\lim _{z \rightarrow 0}|z|^{\alpha} \lambda(z) \cdot z \partial u(z)=-\frac{\alpha}{2} l_{0,0},
$$

which is a real number, hence $l_{1,0}=\overline{l_{0,1}}=l_{0,1}$. Note that

$$
\bar{\partial}^{n} \lambda(z)=\sum_{j=0}^{n-1}\left(\begin{array}{c}
n-1 \\
j
\end{array}\right) \bar{\partial}^{n-j} u(z) \bar{\partial}^{j} \lambda(z),
$$

then by induction

$$
l_{n, 0}=l_{0, n}
$$


From (ii) in Theorem 3.2, we know $\lim _{z \rightarrow 0} \bar{z}^{m} z^{n} \bar{\partial}^{m} \partial^{n} u(z)=0$. This means that we can write $l_{m, n}$ as a sum of the terms not containing any mixed derivatives of $u(z)$,

$$
l_{m, n}=\frac{1}{m ! n !} \lim _{z \rightarrow 0} \sum_{j=0}^{n-1}\left(\begin{array}{c}
n-1 \\
j
\end{array}\right) z^{n-j} \partial^{n-j} u(z)|z|^{\alpha} \bar{z}^{m} z^{j} \bar{\partial}^{m} \partial^{j} \lambda(z) .
$$

By (3.19) and (i) of Theorem 3.2, we have

$$
\begin{aligned}
l_{m, n} & =\sum_{j=0}^{n-1} \lim _{z \rightarrow 0} \frac{1}{m ! n !} \frac{(n-1) !}{j !(n-1-j) !} z^{n-j} \partial^{n-j} u(z) \cdot|z|^{\alpha} \bar{z}^{m} z^{j} \bar{\partial}^{m} \partial^{j} \lambda(z) \\
& =\frac{1}{n} \sum_{j=0}^{n-1} \frac{1}{m !} \frac{1}{j !(n-1-j) !} \lim _{z \rightarrow 0} z^{n-j} \partial^{n-j} u(z) \cdot \lim _{z \rightarrow 0}|z|^{\alpha} \bar{z}^{m} z^{j} \bar{\partial}^{m} \partial^{j} \lambda(z) \\
& =\frac{1}{n} \sum_{j=0}^{n-1} \frac{\alpha(-1)^{n-j}}{2} \frac{1}{m ! j !} \lim _{z \rightarrow 0}|z|^{\alpha} \bar{z}^{m} z^{j} \bar{\partial}^{m} \partial^{j} \lambda(z)=\frac{\alpha}{2 n} \sum_{j=1}^{n-1}(-1)^{n-j} l_{m, j} .
\end{aligned}
$$

Thus

$$
n \cdot l_{m, n}=\frac{\alpha}{2} \sum_{j=0}^{n-2}(-1)^{n-j} l_{m, j}-\frac{\alpha}{2} l_{m, n-1}=-(n-1) l_{m, n-1}-\frac{\alpha}{2} l_{m, n-1} .
$$

Combining with (3.18), we have

$$
\begin{aligned}
l_{m, n} & =\frac{-\frac{\alpha}{2}-n+1}{n} l_{m, n-1}=\cdots=\left(\begin{array}{c}
-\frac{\alpha}{2} \\
n
\end{array}\right) l_{m, 0} \\
& =\left(\begin{array}{c}
-\frac{\alpha}{2} \\
n
\end{array}\right) l_{0, m}=\left(\begin{array}{c}
-\frac{\alpha}{2} \\
n
\end{array}\right)\left(\begin{array}{c}
-\frac{\alpha}{2} \\
m
\end{array}\right) l_{0,0} .
\end{aligned}
$$

When the order $\alpha \in(0,1)$, the sharpness of Theorem 1.1 [16] can be verified by the following result.

Theorem 3.4. For $\lambda$ given by (2.9) with the order $\alpha \in(0,1)$ and $Y(a, b, c)$ given by (3.2), near the origin, the remainder function $v(z)$ satisfies

(i) $\lim _{z \rightarrow 0} \partial v(z)=\frac{a b}{c}$ for $0<\alpha<1 / 2$,

(ii) $\partial v(z)=2 a b+\frac{2 K_{2}^{2} Y(a, b, c)}{K_{1} K_{2}-1} \frac{\bar{z}}{|z|}+O\left(|z|^{\frac{1}{2}}\right)$ near the origin for $\alpha=1 / 2$,

(iii) $\lim _{z \rightarrow 0} z^{n}|z|^{2 \alpha-2} \partial^{n} v(z)=\frac{(-1)^{n-1}(c)_{n-1} K_{2}^{2} Y(a, b, c)}{(1-c)\left(K_{1} K_{2}-1\right)}$ for $n \geq 2$ if $0<\alpha \leq$ $1 / 2$ and $n \geq 1$ if $1 / 2<\alpha<1$, $\geq 1$.

(iv) $\lim _{z \rightarrow 0} \bar{z}^{m} z^{n}|z|^{2 \alpha-2} \bar{\partial}^{m} \partial^{n} v(z)=\frac{(-1)^{n+m}(c)_{n-1}(c)_{m-1} K_{2}^{2} Y(a, b, c)}{K_{1} K_{2}-1}$ for $m, n$

Proof. Since $v(z)=-\beta \log |1-z|+\log K_{3}-\log M(z)$ and

$$
\partial^{n} \log |1-z|=\frac{-(n-1) !}{2(1-z)^{n}},
$$


we can consider $\partial^{n} \log M(z)$ only. From the proof of Theorem 3.2, we know that the $\operatorname{limits}_{\lim _{z \rightarrow 0}} \partial v(z)$ and $\lim _{z \rightarrow 0} z^{n}|z|^{2 \alpha-2} \partial^{n} v(z), n \geq 2$, both depend on the term $\partial^{n} M(z)$. By Lemma 3.1 and (3.13),

$$
\begin{gathered}
\lim _{z \rightarrow 0} \partial v(z)=\lim _{z \rightarrow 0} \frac{\partial M(z)}{M(z)}, \\
\lim _{z \rightarrow 0} z^{n}|z|^{2 \alpha-2} \partial^{n} v(z)=\lim _{z \rightarrow 0} z^{n}|z|^{2 \alpha-2} \frac{\partial^{n} M(z)}{M(z)} .
\end{gathered}
$$

Thus we obtain the four cases above corresponding to ones in Lemma 3.1.

\section{Case $\alpha=1$}

When $\alpha=1$, the formula for $\lambda_{1, \beta, \gamma}(z)$ is to be understood in the limit sense $\lim _{\alpha \rightarrow 1-} \lambda_{\alpha, \beta, \gamma}(z)$. Moreover, when $\alpha=1$ we have

$$
\begin{gathered}
\varphi_{1}(z)=F(a, b, 1 ; z), \quad \varphi_{2}(z)=F(a, b, a+b ; 1-z), \\
K_{1}=-\frac{S}{B}, \quad K_{2}=0, \quad K_{3}=\frac{1}{B(a, b)}:=\frac{1}{B},
\end{gathered}
$$

where

$$
S:=\frac{\pi \sin (\pi(a+b))}{\sin \pi a \sin \pi b}
$$

and then

$$
\lambda_{1, \beta, \gamma}(z)=\frac{1}{|z|} \frac{1}{|1-z|^{\beta}} \frac{K_{3}}{K_{1}\left|\varphi_{1}(z)\right|^{2}+\varphi_{1}(z) \varphi_{2}(\bar{z})+\varphi_{1}(\bar{z}) \varphi_{2}(z)} .
$$

Let $M(z):=K_{1}\left|\varphi_{1}(z)\right|^{2}+\varphi_{1}(z) \varphi_{2}(\bar{z})+\varphi_{1}(\bar{z}) \varphi_{2}(z)$. Then near the origin, the remainder function is

$$
w(z)=-\beta \log |1-z|+\log K_{3}-\log M(z)+\log \log (1 /|z|) .
$$

The assumption of Theorem A and (2.12) show that $a$ and $b$ satisfy

$$
0<a<1, \quad 0<b<1 / 2, \quad 0<a+b<1 \text {. }
$$

The function

$$
2 R-S=4 \Psi(1)-2 \Psi(a)-2 \Psi(b)-\pi \cot \pi a-\pi \cot \pi b
$$

is of special interest where $R:=R(a, b)$ is as in (2.7) and $S$ is given by (4.1). Let $G(x):=2(\Psi(1)-\Psi(x))-\pi \cot \pi x$. For Gamma function $\Gamma(x), 0<x<1$, we have $\Gamma(x) \Gamma(1-x)=\pi / \sin \pi x$. Taking the logarithmic derivatives of both sides leads to

$$
\frac{\Gamma^{\prime}(x)}{\Gamma(x)}-\frac{\Gamma^{\prime}(1-x)}{\Gamma(1-x)}=-\pi \cot \pi x .
$$

Thus

$$
G(x)=2 \Psi(1)-\Psi(x)-\Psi(1-x),
$$


which means $G(x)=G(1-x)$. If $0<x<1$, the fact that the digamma function $\Phi(x)$ is negative and decreasing implies that $G(x)>0$. Since $2 R-S=$ $G(a)+G(b), 2 R-S>0$ for all $a, b$ given by (2.12).

For $\lambda_{1, \beta, \gamma}(z)$ as in (4.2), we can obtain the precise estimate for higher order derivatives of $w(z)$ near the origin.

Theorem 4.1. Suppose that $\lambda(z)$ is given by (4.2) with $\alpha=1, \beta$ and $\gamma$ satisfying the condition in Theorem $A$, and $w(z)$ is the remainder function as in (4.3). Then for $m, n \geq 1$, we have

(i) $\lim _{z \rightarrow 0} z^{n} \log ^{2}(1 /|z|) \partial^{n} w(z)=\frac{(-1)^{n}(n-1) !}{4}(G(a)+G(b))$,

(ii) $\lim _{z \rightarrow 0} z^{n} \bar{z}^{m} \log ^{3}(1 /|z|) \bar{\partial}^{m} \partial^{n} w(z)=\frac{(-1)^{m+n-1}(n-1) !(m-1) !}{4}(G(a)+G(b))$, where the function $G$ is defined by (4.5) and $a, b$ are given by (2.12).

For the proof of Theorem 4.2, we need the following lemma.

Lemma 4.2. For $m \geq 1, n \geq 1$, we have

$$
\begin{gathered}
\partial^{n} \log \log (1 /|z|)=\frac{\mathcal{A}_{n}}{z^{n} \log (1 /|z|)}+\frac{\mathcal{B}_{n}}{z^{n} \log ^{2}(1 /|z|)}+O\left(\frac{1}{z^{n} \log ^{3}(1 /|z|)}\right), \\
\bar{\partial}^{m} \partial^{n} \log \log (1 /|z|)=\frac{\mathcal{C}_{m}}{\bar{z}^{m} z^{n} \log ^{2}(1 /|z|)}+\frac{\mathcal{D}_{m}}{\bar{z}^{m} z^{n} \log ^{3}(1 /|z|)}+O\left(\frac{1}{\bar{z}^{m} z^{n} \log ^{4}(1 /|z|)}\right),
\end{gathered}
$$

where

$$
\begin{gathered}
\mathcal{A}_{n}=\frac{(-1)^{n}}{2}(n-1) ! \\
\mathcal{B}_{n}=\frac{(-1)^{n-1}}{4}(n-1) ! \sum_{j=1}^{n-1} \frac{1}{j} \\
\mathcal{C}_{m}=\frac{(-1)^{m+n-1}}{4}(m-1) !(n-1) ! \\
\mathcal{D}_{m}=\frac{(-1)^{m+n}}{4}(m-1) !(n-1) !\left(\sum_{j=1}^{n-1} \frac{1}{j}+\sum_{j=1}^{m-1} \frac{1}{j}\right) .
\end{gathered}
$$

Proof. By induction we know that

$$
\begin{aligned}
\partial^{n} \log \log (1 /|z|) & =\sum_{j=1}^{n} \frac{C_{j}^{(n)}}{z^{n} \log ^{j}(1 /|z|)}, \\
\bar{\partial}^{m} \partial^{n} \log \log (1 /|z|) & =\sum_{j=1}^{m} \frac{C_{j}^{(m, n)}}{\bar{z}^{m} z^{n} \log ^{j+1}(1 /|z|)}
\end{aligned}
$$

with constants $C_{j}^{(n)}$ and $C_{j}^{(m, n)}, 1 \leq j \leq m, 1 \leq j \leq n$. As for the pure derivative $\partial^{n} \log \log (1 /|z|)$, set $\mathcal{A}_{n}:=C_{1}^{(n)}$ and $\mathcal{B}_{n}:=C_{2}^{(n)}$, hence

$$
\partial^{n} \log \log (1 /|z|)=\frac{\mathcal{A}_{n}}{z^{n} \log (1 /|z|)}+\frac{\mathcal{B}_{n}}{z^{n} \log ^{2}(1 /|z|)}+\sum_{j=3}^{n} \frac{C_{j}^{(n)}}{z^{n} \log ^{j}(1 /|z|)},
$$


then the following recurrent relations hold,

$$
\mathcal{A}_{1}=-\frac{1}{2}, \quad \mathcal{B}_{1}=0, \quad \mathcal{A}_{n}=-(n-1) \mathcal{A}_{n-1}, \quad \mathcal{B}_{n}=-(n-1) \mathcal{B}_{n-1}+\frac{1}{2} \mathcal{A}_{n-1} .
$$

Thus we obtain (4.6) and (4.7). For the mixed derivative case, we fix $n$ and set $\mathcal{C}_{m}:=C_{1}^{(m, n)}, \mathcal{D}_{m}:=C_{2}^{(m, n)}$. Then by induction we have

$\mathcal{C}_{1}=\frac{1}{2} \mathcal{A}_{n}, \quad \mathcal{D}_{1}=\mathcal{B}_{n}, \quad \mathcal{C}_{m}=-(m-1) \mathcal{C}_{m-1}, \quad \mathcal{D}_{m}=-(m-1) \mathcal{D}_{m-1}+\mathcal{C}_{m-1}$.

Therefore (4.8) and (4.9) hold.

Proof of Theorem 4.1. For the remainder function $w(z)$ given by (4.3), we discuss $\frac{\partial^{n} M(z)}{M(z)}, n \geq 1$ at first. Note that

$$
\begin{aligned}
& \partial^{n} \varphi_{1}(z)=\frac{(a)_{n}(b)_{n}}{n !} F(a+n, b+n, n+1 ; z), \\
& \partial^{n} \varphi_{2}(z)=(-1)^{n} \frac{(a)_{n}(b)_{n}}{(a+b)_{n}} F(a+n, b+n, a+b+n ; 1-z),
\end{aligned}
$$

then

$$
\partial^{n} \varphi_{1}(0)=\frac{(a)_{n}(b)_{n}}{n !},
$$

and near the origin, by $(2.5)$ we have

$$
\partial^{n} \varphi_{2}(z)=\frac{(a)_{n}(b)_{n}}{(a+b)_{n}} \frac{(-1)^{n}}{z^{n}} F(b, a, a+b+n ; 1-z) .
$$

Property (2.4) shows that

$$
F(b, a, a+b+n ; 1-z)=\frac{\Gamma(a+b+n) \Gamma(n)}{\Gamma(a+n) \Gamma(b+n)}+O(|z| \log |z|)
$$

near the origin. Thus

$$
\begin{aligned}
\partial^{n} \varphi_{2}(z) & =\frac{(-1)^{n}}{z^{n}} \frac{(a)_{n}(b)_{n}}{(a+b)_{n}}\left(\frac{\Gamma(a+b+n) \Gamma(n)}{\Gamma(a+n) \Gamma(b+n)}+O(|z| \log |z|)\right) \\
& =\frac{(-1)^{n}(n-1) !}{B z^{n}}+O\left(\frac{\log |z|}{|z|^{n-1}}\right) .
\end{aligned}
$$

By (2.5) we know

$$
\varphi_{2}(z)=\frac{1}{B}\left(\log \frac{1}{z}+R\right)(1+O(z)) .
$$

From (4.10), (4.11) and (4.12), we obtain

$$
\begin{aligned}
\partial^{n} M(z) & =\left(K_{1} \varphi_{1}(\bar{z})+\varphi_{2}(\bar{z})\right) \partial^{n} \varphi_{1}(z)+\varphi_{1}(\bar{z}) \partial^{n} \varphi_{2}(z) \\
& =\frac{(a)_{n}(b)_{n}}{B n !} \log \frac{1}{\bar{z}}+\frac{(-1)^{n}(n-1) !}{B z^{n}}+O\left(\frac{\log |z|}{|z|^{n-1}}\right)
\end{aligned}
$$

and

$$
M(z)=K_{1} \varphi_{1}(\bar{z}) \varphi_{1}(z)+\varphi_{2}(\bar{z}) \varphi_{1}(z)+\varphi_{1}(\bar{z}) \varphi_{2}(z)
$$




$$
=\frac{2 \log (1 /|z|)}{B}\left(1+\frac{2 R-S}{2 \log (1 /|z|)}+O(|z|)\right)
$$

near the origin. Then

$$
\begin{aligned}
\frac{\partial^{n} M(z)}{M(z)} & =\partial^{n} M(z) \frac{B}{2 \log (1 /|z|)}\left(1-\frac{2 R-S}{2 \log (1 /|z|)}+O(|z|)\right) \\
(4.13) \quad & =\frac{(-1)^{n}(n-1) !}{2 z^{n} \log (1 /|z|)}-\frac{(-1)^{n}(n-1) !(G(a)+G(b))}{4 z^{n} \log ^{2}(1 /|z|)}+O\left(\frac{1}{|z|^{n-1}}\right) .
\end{aligned}
$$

If we note that

$$
\begin{aligned}
\bar{\partial}^{m} \partial^{n} M(z)= & \left(K_{1} \bar{\partial}^{m} \varphi_{1}(\bar{z})+\bar{\partial}^{m} \varphi_{2}(\bar{z})\right) \partial^{n} \varphi_{1}(z)+\bar{\partial}^{m} \varphi_{1}(\bar{z}) \partial^{n} \varphi_{2}(z) \\
= & \frac{(-1)^{m}(m-1) !}{B \bar{z}^{m}} \frac{(a)_{n}(b)_{n}}{n !}+\frac{(-1)^{n}(n-1) !}{B z^{n}} \frac{(a)_{m}(b)_{m}}{m !} \\
& +O\left(\frac{\log |z|}{|z|^{m-1}}\right)+O\left(\frac{\log |z|}{|z|^{n-1}}\right)
\end{aligned}
$$

the same technique leads to

$$
\frac{\bar{\partial}^{m} \partial^{n} M(z)}{M(z)}=O\left(\frac{1}{|z|^{\tau} \log (1 /|z|)}\right)
$$

where $\tau=\max \{m, n\}<m+n$.

Now we consider derivatives of $w(z)$. In the pure derivative case,

$$
\partial^{n} w(z)=\frac{\beta(n-1) !}{2(1-z)^{n}}-\partial^{n} \log M(z)+\partial^{n} \log \log (1 /|z|) .
$$

Note that $\partial^{n} \log M(z)$ is the linear combination of finitely many terms in the form

$$
\prod_{j=1}^{N} \frac{\partial^{n_{j}} M(z)}{M(z)}
$$

for $1 \leq N \leq n$ and $\sum_{j=1}^{k} n_{j}=n$. When $N=1$, term (4.16) is equal to $\frac{\partial^{n} M(z)}{M(z)}$. By induction we know the coefficient of $\frac{\partial^{n} M(z)}{M(z)}$ in $\partial^{n} \log M(z)$ is always 1 . Note that the term contains $z^{-n} \log ^{-1}(1 /|z|)$ in $\partial^{n} \log M(z)$ only appears in $\partial^{n} M(z) / M(z)$, and then (4.6), (4.13), (4.15) show that this term is canceled in $\partial^{n} w(z)$. Now we look at the term which contains $z^{-n} \log ^{-2}(1 /|z|)$ in (4.15), while the higher power terms in $\frac{\partial^{n} M(z)}{M(z)}$ are ignored for a moment. For (4.16), estimate (4.13) shows that

$$
\prod_{j=1}^{N} \frac{\partial^{n_{j}} M(z)}{M(z)}=O\left(\frac{1}{z^{n} \log ^{N}(1 /|z|)}\right) \text { for } n=\sum_{j=1}^{N} n_{j},
$$


therefore, to generate the $z^{-n} \log ^{-2}(1 /|z|)$ term, $N$ is at most 2 , thus the $z^{-n} \log ^{-2}|z|$ term of $\partial^{n} \log M(z)$ only appears in

$$
\frac{\partial^{n} M}{M}-\frac{1}{2} \sum_{j=1}^{n-1}\left(\begin{array}{l}
n \\
j
\end{array}\right) \frac{\partial^{j} M \partial^{n-j} M}{M^{2}}
$$

For every $1 \leq j \leq n-1$, letting $n=1$ in (4.13), we have

$$
\begin{aligned}
\frac{\partial^{j} M \partial^{n-j} M}{M^{2}} & =\frac{\partial^{j} M}{M} \cdot \frac{\partial^{n-j} M}{M} \\
& =\frac{(-1)^{n}(j-1) !(n-j-1) !}{4 z^{n} \log ^{2}|z|}+O\left(\frac{1}{|z|^{n} \log ^{3}(1 /|z|)}\right) .
\end{aligned}
$$

Let $k_{n}$ be the coefficient of $z^{-n} \log ^{-2}|z|$ in $\sum_{j=1}^{n-1}\left(\begin{array}{c}n \\ j\end{array}\right)\left(\partial^{j} M \partial^{n-j} M / M^{2}\right)$. Then (4.17) leads to

$$
\begin{aligned}
k_{n} & =\sum_{j=1}^{n-1}\left(\begin{array}{l}
n \\
j
\end{array}\right) \frac{(-1)^{n}(j-1) !(n-j-1) !}{4}=\frac{(-1)^{n}(n-1) !}{4} \sum_{j=1}^{n-1} \frac{n}{j(n-j)} \\
& =\frac{(-1)^{n}(n-1) !}{4} \sum_{j=1}^{n-1}\left(\frac{1}{j}+\frac{1}{n-j}\right)=\frac{(-1)^{n}(n-1) !}{2} \sum_{j=1}^{n-1} \frac{1}{j}=-2 \mathcal{B}_{n},
\end{aligned}
$$

where $\mathcal{B}_{n}$ is given by (4.7). From (4.7) and (4.18), we obtain

$$
\begin{aligned}
\lim _{z \rightarrow 0} z^{n} \log ^{2}(1 /|z|) \partial^{n} w(z) & =\frac{(-1)^{n}(n-1) !(G(a)+G(b))}{4}+\frac{1}{2} k_{n}+\mathcal{B}_{n} \\
& =\frac{(-1)^{n}(n-1) !}{4}(G(a)+G(b))
\end{aligned}
$$

thus (i) holds.

For the mixed derivative,

$$
\bar{\partial}^{m} \partial^{n} w(z)=-\bar{\partial}^{m} \partial^{n} \log M(z)+\bar{\partial}^{m} \partial^{n} \log \log (1 /|z|) .
$$

It is known that $M(z)=M(\bar{z})$ and $\bar{\partial}^{m} \partial^{n} \log M(z)=\overline{\bar{\partial}^{n} \partial^{m} \log M(z)}$. Hence without loss of generality we may assume $m \leq n$. Note that in $\bar{\partial}^{m} \partial^{n} \log M(z)$, the term $\bar{z}^{-m} z^{-n} \log ^{-2}(1 /|z|)$ with coefficient $(-1)^{m+n}(n-1) !(m-1) ! / 4$ only occurs in $\bar{\partial}^{m} M \partial^{n} M / M^{2}$, from (4.8) we know that there is no term contains $\bar{z}^{-m} z^{-n} \log ^{-2}(1 /|z|)$ left in the expression for $\bar{\partial}^{m} \partial^{n} w(z)$. Therefore the coefficient of $z^{-n} \bar{z}^{-m} \log ^{-3}(1 /|z|)$ is desired. The term contains $z^{-n} \bar{z}^{-m} \log ^{-3}(1 /|z|)$ is the product of at most three terms in the forms of $\partial^{n_{j}} M / M$ or $\bar{\partial}^{m_{j}} M / M$. By (4.14) and (4.13), the term $z^{-n} \bar{z}^{-m} \log ^{-3}(1 /|z|)$ in $\bar{\partial}^{m} \partial^{n} \log M(z)$ only appears in

$$
-\frac{\bar{\partial}^{m} M \partial^{n} M}{M^{2}}+\frac{\bar{\partial}^{m} M}{M} \sum_{j=1}^{n-1}\left(\begin{array}{l}
n \\
k
\end{array}\right) \frac{\partial^{j} M \partial^{n-j} M}{M^{2}}+\frac{\partial^{n} M}{M} \sum_{j=1}^{m-1}\left(\begin{array}{c}
m \\
k
\end{array}\right) \frac{\bar{\partial}^{j} M \bar{\partial}^{m-j} M}{M^{2}}
$$


for $m \geq 1, n \geq 1$. Then, similar to $k_{n}$ given by (4.18),

with

$$
\bar{\partial}^{m} \partial^{n} \log M(z)=\frac{t_{m, n}}{z^{n} \bar{z}^{m} \log ^{3}(1 /|z|)}+O\left(\frac{1}{|z|^{m+n+1} \log ^{3}(1 /|z|)}\right)
$$

$$
t_{m, n}=\left(G(a)+G(b)+\sum_{j=1}^{n-1} \frac{1}{j}+\sum_{j=1}^{m-1} \frac{1}{j}\right) \frac{(-1)^{m+n}(m-1) !(n-1) !}{4} .
$$

Thus for $m \geq 1, n \geq 1$, by (4.9) and (4.19), we obtain

$$
\begin{aligned}
\lim _{z \rightarrow 0} z^{n} \bar{z}^{m} \log ^{3}(1 /|z|) \bar{\partial}^{m} \partial^{n} w(z) & =-t_{m, n}+\mathcal{D}_{m} \\
& =\frac{(-1)^{m+n-1}(n-1) !(m-1) !}{4}(G(a)+G(b))
\end{aligned}
$$

This completes the proof.

Remark. Theorem 4.1 verifies the sharpness of Theorem 1.1 in [16] when the order of the singularity is 1 .

When $\alpha=1$, there is an analogue of Theorem 3.2 and Theorem 3.3, see [16], also [3] and [7]. Here we list them as follows without proof.

Theorem 4.3. For $\lambda(z):=\lambda_{1, \beta, \gamma}(z)$ as in (4.2), let $u(z):=\log \lambda(z)$. Then for $m, n \geq 1$,

(i) $\lim _{z \rightarrow 0} z^{n} \partial^{n} u(z)=\frac{1}{2}(-1)^{n}(n-1) !=\lim _{z \rightarrow 0} \bar{z}^{n} \bar{\partial}^{n} u(z)$,

(ii) $\lim _{z \rightarrow 0} \bar{z}^{m} z^{n} \log ^{2}(1 /|z|) \bar{\partial}^{m} \partial^{n} u(z)=\frac{(-1)^{n+m}(n-1) !(m-1) !}{4}$.

Theorem $\mathbf{C}([15])$. For $m, n \geq 0, \alpha=1$ and $\lambda(z)$ as in (4.2), the limit

$$
l_{m, n}^{\prime}:=\frac{1}{n ! m !} \lim _{z \rightarrow 0}|z| \log (1 /|z|) \bar{z}^{m} z^{n} \bar{\partial}^{m} \partial^{n} \lambda(z)
$$

exists. Moreover, the numbers $l_{m, n}^{\prime}$ satisfy the following.

(i) $l_{0,0}^{\prime}:=\lim _{z \rightarrow 0}|z| \log (1 /|z|) \lambda(z)=\frac{1}{2}$,

(ii) $l_{m, n}^{\prime}=\frac{1}{2}\left(\frac{-1}{n}\right)\left(\begin{array}{c}-\frac{1}{2} \\ m\end{array}\right)$,

(iii) $l_{m, n}^{\prime}=l_{n, m}^{\prime}$.

Acknowledgement. I would like to thank Prof. Toshiyoki Sugawa for his helpful comments, suggestion and encouragement. I also want to thank Rintaro Ohno for his considerable time reading through each draft. I am grateful to the referees for their comments.

\section{References}

[1] M. Abramowitz and I. A. Stegun, Handbook of Mathematical Functions With Formulas, Graphs and Mathematical Tables, Dover, New York, 1964.

[2] G. D. Anderson, T. Sugawa, M. K. Vamanamurthy, and M. Vuorinen, Hypergeometric functions and hyperbolic metric, Comput. Methods Funct. Theory 9 (2009), no. 1, 269284 . 
[3] B. T. Gill and T. H. MacGregor, Derivatives of the hyperbolic density near an isolated boundary point, Rocky Mountain J. Math. 36 (2006), no. 6, 1873-1884.

[4] M. Heins, On a class of conformal metrics, Nagoya Math. J. 21 (1962), 1-60.

[5] D. Kraus and O. Roth, Conformal metrics, Ramanujan Math. Society, Lecture Notes Series 19 (2013), 41-83.

[6] - On the isolated singularities of the solutions of the Gaussian curvature equation for nonnegative curvature, J. Math. Anal. Appl. 345 (2008), no. 2, 628-631.

7] _ The behaviour of solutions of the Gaussian curvature equation near an isolated boundary point, Math. Proc. Cambridge Philos. Soc. 145 (2008), no. 3, 643-667.

[8] D. Kraus, O. Roth, and St. Ruscheweyh, A boundary version of Ahlfors' lemma, locally complete conformal metrics and conformally invariant reflection principles for analytic maps, J. Anal. Math. 101 (2007), 219-256.

[9] D. Kraus, O. Roth, and T. Sugawa, Metrics with conical singularities on the sphere and sharp extensions of the theorems of Landau and Schottky, Math. Z. 267 (2011), no. 3-4, 851-868.

[10] D. Minda, The density of the hyperbolic metric near an isolated boundary point, Complex Variables Theory Appl. 32 (1997), no. 4 331-340.

[11] J. Nitsche, Über die isolierten Singularitäten der Lösungen von $\Delta u=e^{u}$, Math. Z. 68 (1957), 316-324.

[12] E. Picard, De l'integration de l'équation differentielles $\Delta u=e^{u}$ sur une surface de Riemann fermée, J. Reine Angew. Math. 130 (1905), 243-258.

[13] M. Troyanov, Prescribing curvature on compact surfaces with conical singularities, Trans. Amer. Math. Soc. 324 (1991), no. 2, 793-821.

[14] S. T. Yau, A general Schwarz lemma for Kähler manifolds, Amer. J. Math. 100 (1978), no. $1,197-203$.

[15] T. Zhang, Variants of Ahlfors' lemma and properties of the logarithmic potentials, Interactions between real and complex analysis, 33-47, Sci. Technics Publ. House, Hanoi, 2012.

[16] _ A note on the asymptotic behavior of conformal metrics with negative curvatures near isolated singularities, (submitted) arXiv: 1304.2004 [math.CV].

Graduate School of Information Sciences

TOHOKU UNIVERSITY

Aoba-Ku, Sendai 980-8579, Japan

E-mail address: zhang@ims.is.tohoku.ac.jp 